\title{
Risk perception in epidemic modeling
}

\author{
Franco Bagnoli \\ Department of Energy, University of Florence \\ Via S. Marta,3 I-50139 Firenze, Italy \\ also CSDC and INFN, sez. FirenzÆ \\ Pietro Liò \\ Computer Laboratory, University of Cambridge \\ JJ Thompson Avenue, CB30FD Cambridge UK \\ Luca Sguanci \\ Centro per lo Studio di Dinamiche Complesse (CSDC), \\ University of Florence, Via G. Sansone 1, Sesto Fiorentino (FI)
}

(Dated: May 30, 2018)

\begin{abstract}
We investigate the effects of risk perception in a simple model of epidemic spreading. We assume that the perception of the risk of being infected depends on the fraction of neighbors that are ill. The effect of this factor is to decrease the infectivity, that therefore becomes a dynamical component of the model. We study the problem in the mean-field approximation and by numerical simulations for regular, random and scale-free networks. We show that for homogeneous and random networks, there is always a value of perception that stops the epidemics. In the "worst-case" scenario of a scale-free network with diverging input connectivity, a linear perception cannot stop the epidemics; however we show that a non-linear increase of the perception risk may lead to the extinction of the disease. This transition is discontinuous, and is not predicted by the mean-field analysis.
\end{abstract}

\section{INTRODUCTION}

In spring 2006, the potential threat of bird flu dominated headlines in UK newspapers. On 26 March 2006 The Sun has called it "the day we all dreaded", while the Guardian says avian flu is "almost certain to spread to wild birds across the UK". The Daily Telegraph adds that the most likely human victims will be poultry farmers, who will be bankrupted. But the Mirror calls for calm, saying people have a better chance of winning the lottery than catching the virus. Interestingly, given a certain amount of clustering of wealth residents and correlation between wealth and readers preference, this would translate into a differently informed neighborhood. When the epidemic is over its peak or other news has just peaked or media has "cried wolf" too many times over unfounded health scares, there is a quick drop in the attention to that disease (something similar is reported nowadays for HIV). In other parts of the world, for example Indonesia, a country with 18000 islands, people reacted differently to the bird flu epidemics. Despite awareness campaigns in the media and even door-to-door visits in some of the islands, many Indonesians remained oblivious to the dangers of being in contact with diseased birds, and aware of the need to inform the authorities and implement a cull. Note that awareness campaigns, such as during the SARS epidemics, are expensive and may result in culling, reductions in commerce, travels and tourism. The media hype over epidemics threat has a close sim-

*Electronic address: franco.bagnoli@unifi.it ilarity in how worried or fatalist, resilient, skeptical or cheeky may be friends and neighborhood. Therefore, the individual perception of the risk of becoming infected is a key factor influencing the spreading of an epidemics and, toward realistic inference, epidemiological models should incorporate such parameter [1].

In order to investigate the effect of risk perception in influencing the spreading of a disease, let us start from simple, yet meaningful models, such SIS or SIR ones. These models are defined on a network where individuals or groups of individuals corresponds to the nodes and links represent social contacts and relationships among them. Most of classical studies used either a regular lattice, or a random one. Both of those choices are characterized by a well defined value of the mean connectivity $\langle k\rangle$, and small variance $\left\langle k^{2}\right\rangle-\langle k\rangle^{2}$. As shown by Watts and Strogatz 2], the simple rewiring of a small fraction of links in an otherwise regular lattice results in a sudden lowering of the diameter of the graph, without affecting the average connectivity or the degree of clustering. This small world effect manifests itself in a dramatic shortage of the distance between any two individuals, almost without affecting the local perception of the network of contacts. The consequences for epidemics spreading are important: just a few long-distance connections may promote the spreading of a disease in rural areas, whereby an epidemic would otherwise diffuse very slowly.

However, the investigations of social networks have shown that they are quite different from regular and random graphs [3, 4]. The probability distribution of contacts often exhibits a power-law behavior $\left(P(k) \propto k^{-\gamma}\right)$, with an exponent $\gamma$ between two and three [5, 6]. This distribution is characterized by a relatively large number 
of highly connected hubs, which are presumably responsible for epidemics spreading. Moreover, such distributions have a diverging second moment $\left\langle k^{2}\right\rangle$ for $\gamma \leq 3$ and a diverging average connectivity $\langle k\rangle$ for $\gamma \leq 2$.

The influence of the connectivity on the spreading dynamics is well outlined by a simple mean-field analysis. Let us consider for the moment a tree with fixed connectivity $k$. In a SIS model with immediate recovery dynamics, a single infected individual may infect up to $k$ neighbors [7], each one with probability $\tau$. The temporal behavior of the mean fraction $c$ of infected individuals is given by

$$
c^{\prime}=\sum_{s=1}^{k}\left(\begin{array}{l}
k \\
s
\end{array}\right) c^{s}(1-c)^{k-s}\left(1-(1-\tau)^{s}\right),
$$

where $c \equiv c(t), c^{\prime} \equiv c(t+1)$ and the sum runs over the number $s$ of infected individuals. The basic reproductive ratio $R_{0}$ [8] is simply given by $R_{0}=k \tau$, so that the epidemic threshold $R_{0}=1$ corresponds to $\tau_{c}=1 / k$. This means that for a fixed connectivity, only diseases with an infectivity less than $1 / k$ do not spread.

In heterogeneous networks (nodes with different connectivity) the mean field analysis, reported in Section II gives $\tau_{c}=\left\langle k^{2}\right\rangle /\langle k\rangle$. In the case $\left\langle k^{2}\right\rangle \simeq\langle k\rangle^{2}, \tau_{c}$ is again equal to $1 /\langle k\rangle$.

In summary, the result is that on very non homogeneous networks, with diverging second moment $\left\langle k^{2}\right\rangle$ (and even worse on those with diverging average connectivity $\langle k\rangle$ ), a disease will always spread regardless of its intrinsic morbidity 9 ].

This result can be modified by the assortativity degree of the network and by the presence of loops, not considered in tyhe mean-field analysis. In networks with assortative connections (hubs are preferentially connected to other hubs), it may happen that epidemics spread for any finite infectivity even when the second moment is not diverging [10, 11], while for disassortative networks the reverse is true, epidemics may be stopped by lowering the infectivity with random vaccination campaigns, even in the presence of a diverging second moment [10]. This is particularly evident in networks lacking the small-world property (consequence of high disassortativity) [12, 13].

In small-world networks with diverging second moment, it is quite difficult to stop an epidemics. The most common recipes are vaccination campaigns (removal of nodes) or modification of the social structure (removal of links), that mathematically corresponds to site and bond percolation problems. To be efficient, a vaccination camping must be targeted to hubs, either directly [3] or implicitly, for instance by exploiting the fact that hubs are the most probable neighbors of many nodes [14].

The modification of the social structure can be obtained by cohercitive methods (quarantine, etc.) or by rising alerts so to modify travelling and business patterns, but this option may be so expensive that the amount of money put into restoring the previous situation may exceed that used to cure ill people [15].
However, epidemics in modern world are relatively uncommon, and most of them are stopped quite easily in spite of the presence of high network connectivity. The existence of an epidemic threshold on such networks has motivated the investigation of the effects of connectivitydependent infectivity [16, 17, 18]. In this latter case, most of investigations have been performed using meanfield techniques, thus disregarding the presence of loops.

Loops are irrelevant at and near the percolation threshold [19], and therefore one can threat the network as a tree in these conditions. However, for processes evolving on percolating networks, this assumption may not hold.

At present, the basic models used do not take into consideration the knowledge that all human beings have nowadays about the mechanisms of diffusion of diseases. In fact, even in the absence of vaccination campaigns, a disease that manifests itself in a visible way induces modifications in the social network: lower frequency of contacts (usage of mass transportation systems), higher level of personal hygiene, prevention measures (masks), etc. Indeed, recent works stress the importance of using a time-dependent bare infectivity to reproduce real patterns of epidemics [20, 21, 22, 23].

Viruses with high mutation rates (like computer viruses) follow a dynamics which is more similar to SIS than to SIR 24], even in the presence of immunization. On the other hand, the origin of vaccination come from cross-immunization conferred by strains with lower pathogenicity.

We shall study here a SIS model in which the bare infectivity of the spreading agent is modulated by a term that tries to model the effects of the perception of the risk of being infected.

We assume that this perception is just an increasing function of the fraction of ill people in the neighborhood, given that the illness presents visible symptoms. This assumption is modeled after the heuristicsystematic information-processing model [25], that simply states that attitudes are formed and modified as people gain information about a process. In the absence of explicit alarm or communication, the only way of gaining this information is though examination of people in the neighborhood. Individuals can process information in two ways: either heuristically, using simple an semiunconscious schemes, or carefully examining them in a rational way. Investigations about the effects of advertisements, especially those exploiting fear, show that the first way is rather common and predictable, except in the limit of high level of fear, that may induce repulsion towards the brand, or very low level, that may trigger the reflexive mechanism and a more careful evaluation of the message.

In this work we simply assume that the local information (not enhanced by alarms) about the incidence of the illness translates into a lowering of the infection probability, implementing only the "linear part" of the information-processing model. In principle, is possible to compare the effective susceptibility to infection for 
diseases that manifest themselves in a visible and in an invisible way and test experimentally this hypothesis.

In our model, the infectivity is a dynamical quantity. Although the idea of modulating the infectivity of the infection process is not new, it is generally studied (mostly in the mean-field approximation) as as a function of time [20, 21, 22, 23], of connectivity 16, 17] and/or depending on the total infection level [26, 27]. In this latter approach, a nonlinear growing dependence of the infection rate on the total number of infected people may originate bifurcation and chaotic oscillations.

As we shall show in the following, mean-field analysis may not capture the essential phenomena in highly connected networks. Moreover, we study the case of decreasing infection rate with increasing local infection level, that might also induce chaotic oscillations at the meanfield level (See Ref. [28] and Section [II). However, one should consider that chaotic oscillations on networks easily desynchronize, and the resulting "microscopic chaos" is quite different from the synchronous oscillations predicted by mean-field analysis [29], that may nevertheless be observed in lattice models the presence of long-range coupling [30].

We explicitly describe the model in Section II, analyze it using mean-field techniques in Section [II and study numerically its behavior on different types of networks in Section [V] Conclusions and perspectives are drawn in the last section.

\section{THE MODEL}

In this paper we study the dynamics of an infection spreading over a network of $N$ individuals. We use different kinds of networks: regular, with long-range rewiring [2], random and scale-free [5]. The network structure is considered not to depend on the infection level.

Let us denote by $P(k)$ the probability distribution of connectivity $k$. We shall denote by $z=\mu_{1}(P)$ the average connectivity (first moment of the distribution), $z=\langle k\rangle=$ $\sum_{k} k P(k)$, and by $\mu_{2}$ and $\mu_{2}=z^{2}$. The rewiring of the network is performed by starting from a regular lattice in one dimension, detaching a fraction $p$ of links from one end and attaching them to randomly chosen nodes. The regular case is studied numerically in one dimension. Simulations on the rewired network are performed both in the quenched and in the annealed cases.

For random graphs, studied only at the mean-field level, the probability distribution is assumed to be Poissonian,

$$
P(k)=\frac{z^{k} e^{-z}}{k !},
$$

corresponding to drawing $N z$ links at random among the $N$ nodes $\left(\mu_{2}=z\right)$.

The scale-free network that we study numerically is asymmetric: each node $i$ has a certain number $k_{\text {in }}(i)$ of

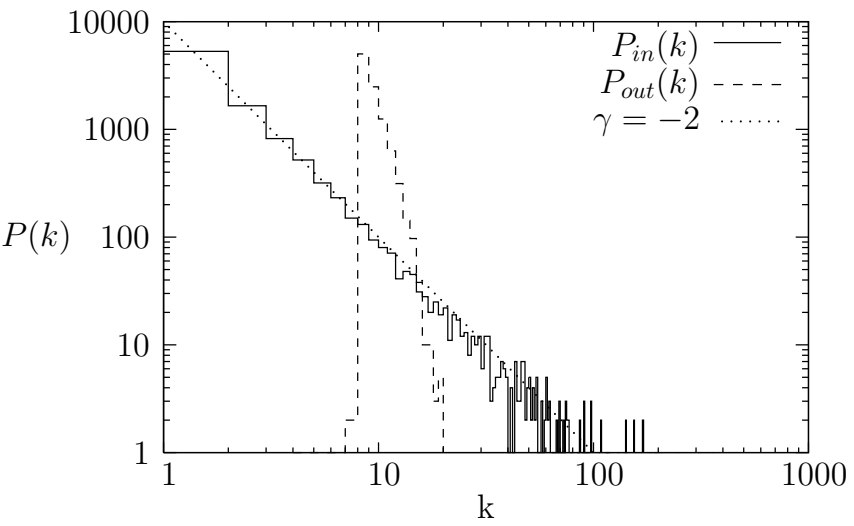

FIG. 1: Distribution of input and output connections for the scale-free network used in simulations.

input contacts and $k_{\text {out }}(i)$ of output ones, and was grown using the following rule.

We start with a ring of $K$ nodes, and we add the other $N-K$ nodes by choosing, for each of them, $K$ connected nodes $j_{n}, n=1, \ldots, K$, with probability $k_{\text {in }}\left(j_{n}\right) / \sum_{l=1}^{N} k_{\text {in }}(l)$ (preferential attachment). The node being attached is added to the inputs of the chosen nodes. We also choose another node at random and add it to the list of input nodes of the new node. This process simulates the growing of a social network in which a new node (a family or an individual) is born from another one (the ones that is added as input of the newborn node) and joins the society according with the popularity of nodes.

Our procedure allows to generate a network that has a power-law distribution of input contacts, $P_{\text {in }}(k) \simeq k^{-\gamma}$, with $\gamma \simeq 2$ (see Figure 1), while the distribution of output connections, $P_{\text {out }}(k)$, is found to be exponentially distributed. This is an interesting feature of the model as the input connections represent the total number of contacts to which an individual is exposed, while the output connections represent the actively pursued contacts, e.g. familiar ones. A customer, for instance, is exposed to a large number of obliged contacts, and may become infected with a large probability. These are considered "input" links. On the other hand, people in a public position is more monitored, and it is not plausible that they can infect a comparable large number of people. Infection is limited to the private sphere, where contacts are more intense. These are the "output" links. We choose this algorithm in order to have a "worst-case" scenario, with an exponent corresponding to a diverging average of input connectivity

We have not studied the case of dynamic dependence of the network on the infection level, however a high-level of infection of a severe disease may surely induce changes in the social network. It is reasonable to assume that, for mild diseases (or diseases considered harmless, like most of computer viruses), the social network is not affected and only the level of prevention is increased.

In the present paper we assume the effects of the in- 


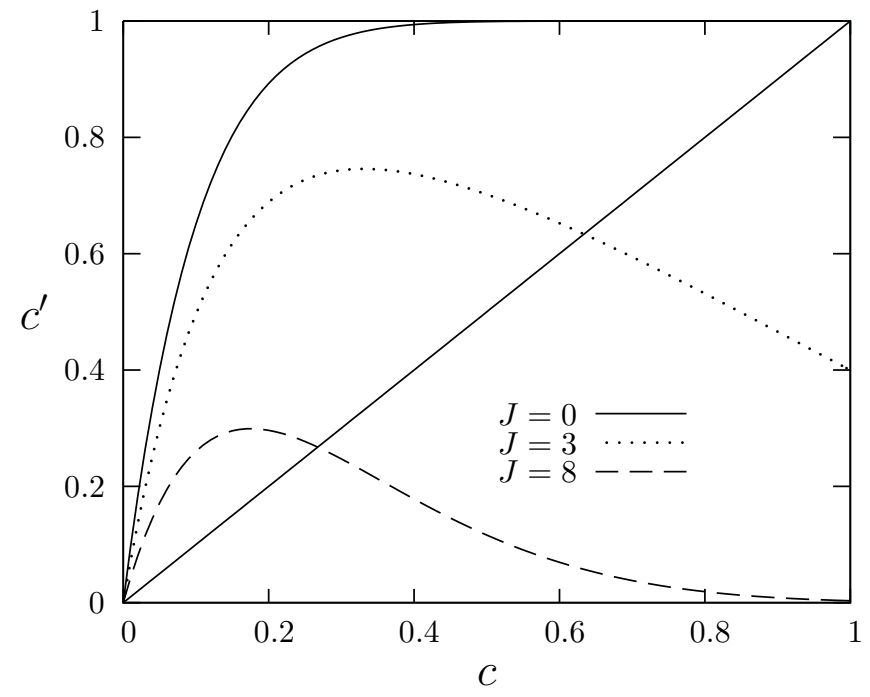

FIG. 2: Mean field return map for fixed connectivity $z=$ 10 , parameters $H=0, \alpha=1, \tau=1$ and varying values of precaution level $J$. The effect of risk perception $(J)$ is to lower the infectivity at high concentrations of infected individuals.

fection to be immediately visible, with no latency nor "hidden infectivity". We also assume as temporal unit the time required to recover from illness without immunization and thus we explore the case of a SIS dynamics.

An individual can be infected separately by each of his neighbors with a probability $\tau$ per unit of time (see Eq. (11)).

We model the effects of the perception of the risk of being infected replacing the bare infection probability $\tau$ with $\tau I(s, k)$,

$$
I(s, k)=\exp \left\{-\left[H+J\left(\frac{s}{k}\right)^{\alpha}\right]\right\}
$$

where $k$ is the number of neighbors of a given site and $s$ is the number of them that are ill.

We assume the perception of the risk of being infected to depend on the fraction of infected individuals among the neighbors, $s / k$, on the level of precaution measures adopted, $J$, and on the use of special prophylaxis, $\alpha \leq$ 1. The quantity $H$ models a global influence over the population, either alarm of broadcasting media news, in which case it could depend on the average level of the infection. Its effect is that of reducing the bare infectivity $\tau$, so in the following we only consider the case $H=0$. For the moment, we consider $\alpha=1$; the role of this parameter will be clear in the following. Differently from Ref [17], in our model the infectivity is not exclusively related to the connectivity.

The mean-field return map (for fixed connectivity $z$ ) is shown in Figure 2. The effect of the introduction of risk perception is evident: for high concentrations of infected individuals the probability of being infected is diminished. Therefore, while for $J=0$ and $z>1$ there is only one stable fixed point $c=1$ (all individuals infected), by increasing $J$ one can have stable fixed points $c<1$, limit cycles and even chaotic behavior [28].

\section{MEAN FIELD ANALYSIS}

The simplest mean-field approximation of the evolution of disease on a network consists in neglecting correlations among variables. This is essentially equivalent in considering the evolution on a tree, i.e., in assuming the absence of loops.

Let us denote with $c_{k}=c_{k}(t)$ the probability of having an infected site of degree $k$ (with $k$ connections) at time $t$, and with $c_{k}^{\prime}=c_{k}(t+1)$ the probability at a subsequent time step.

The mean evolution of the system is generically given by

$$
c_{k}^{\prime}=\sum_{\mathcal{C}_{k}} P_{C}\left(k \mid \mathcal{C}_{k}\right) P_{I}\left(k, \mathcal{C}_{k}\right) P_{H}\left(\mathcal{C}_{k}\right),
$$

where $\mathcal{C}_{k}$ indicates the local configuration (degrees and healthy status) at time $t$ around a site of degree $k$. $P_{H}\left(\mathcal{C}_{k}\right)$ is the probability of occurrence of the healthy status of such configuration, $P_{C}\left(k \mid \mathcal{C}_{k}\right)$ is the probability that the local configuration is connected to the site under examination, and $P_{I}\left(k, \mathcal{C}_{k}\right)$ is the probability that the disease propagates in one time step from $\mathcal{C}_{k}$ to the site.

In our case, the local configuration is given by a set of $k$ nodes, of degree $\left(n_{1}, n_{2}, \ldots, n_{k}\right)$, and status $\left(s_{1}, s_{2}, \ldots, s_{k}\right)$, where $s_{i}=0$ (1) indicates that the site $i$ is healthy (ill). Thus $\mathcal{C}_{k}=\left(n_{i}, s_{i}\right)_{i=1}^{k}$ and $P_{H}\left(\mathcal{C}_{k}\right)=\prod_{i=1}^{k} c_{n_{i}}^{s_{i}}\left(1-c_{n_{i}}\right)^{1-s_{i}}$ since we assume decorrelation among sites.

$P_{C}\left(k \mid \mathcal{C}_{k}\right)$ depends on the assortativity of the network. Let us define $P_{L}(n \mid k)$ as the probability that a site of degree $k$ is attached to a link connected to a site of degree $n$. $P_{L}(n \mid k)$ is computed in an existing network as the number of links that connects sites of degree $n$ and $k$, divided by the total number of links that are connected to sites of degree $k$, and $\sum_{n} P_{L}(n \mid k)=1$. The detailed balance condition gives $k P_{L}(n \mid k) P(k)=n P_{L}(k \mid n) P(n)$. For non-assortative networks, $P_{L}(n \mid k)=\phi(n)$, and summing over the detailed balance condition one gets $P_{L}(n \mid k)=$ $n P(n) / z$, where $z$ is the average number of links per node, $z=\sum_{k} k P(k)$. Assuming again a decorrelated network, we have

$$
P_{C}\left(k \mid \mathcal{C}_{k}\right)=\prod_{i=1}^{k} P_{L}\left(n_{i} \mid k\right)=\prod_{i=1}^{k} \frac{n_{i} P\left(n_{i}\right)}{z}
$$

for non-assortative networks.

$P_{I}\left(k, \mathcal{C}_{k}\right)$ is the infection probability. In the case without risk perception, it is

$$
P_{I}\left(k, \mathcal{C}_{k}\right)=\left(1-(1-\tau)^{s}\right),
$$

where $s=\sum_{i} s_{i}$. The risk perception is modeled by replacing $\tau$ with $\tau \exp (-J s / k)$, which makes the equations 
hard to be managed analytically except in the limit of vanishing infection probability $c_{k} \rightarrow 0$, for which only the case $s=1$ is relevant. We shall consider this point later.

Putting all together, one gets

$$
\begin{aligned}
c_{k}^{\prime}= & \sum_{\substack{n_{1}, n_{2}, \ldots, n_{k} \\
s_{1}, s_{2}, \ldots, s_{k}}}\left(\prod_{i=1}^{k} P_{L}\left(n_{i} \mid k\right) c_{n_{i}}^{s_{i}}\left(1-c_{n_{i}}\right)^{1-s_{i}}\right) \\
& \cdot\left(1-\prod_{i}(1-\tau)^{s_{i}}\right) .
\end{aligned}
$$

Using the relation

$$
\sum_{x_{1}, x_{2}, \ldots, x_{k}} \prod_{i} f\left(x_{i}\right)=\left(\sum_{x} f(x)\right)^{k},
$$

we obtain after some simplifications

$$
c_{k}^{\prime}=1-\left(1-\tau \sum_{n} c_{n} P_{L}(n \mid k)\right)^{k} .
$$

This expression could be obtained directly by noticing that $1-c$ is the probability of not being ill, which corresponds to the combined probability of not being infected by any of the $k$ neighbors. Neglecting correlations, these are $k$ independent processes (although they depend on $k$ ). Each of these process is 1 minus the probability of being infected, which is the sum, over all possible degree $n$ of the neighboring node, of the probability that it is ill $\left(c_{n}\right)$ times the probability that is is connected to the node under investigation, $P_{L}(n \mid k)$.

Let us denote by $\bar{c}$ the asymptotic value of $c(t)$. Assuming that the transition between the quiescent $(\bar{c}=0)$ and active $(\bar{c}>0)$ is continuous, its boundary is given by the values of parameters for which $c^{\prime} / c=1$ in the limit $c \rightarrow 0$. In this limit

$$
c_{k}^{\prime} \simeq k \tau \sum_{n} c_{n} P(n \mid k),
$$

and we can now consider the case with risk perception, with $\tau$ replaced by $\tau \exp \left(-J / k^{\alpha}\right)$.

In the case of non-assortative networks,

$$
c_{k}(t+1)=k \frac{\tau}{z} \exp \left(-\frac{J}{k^{\alpha}}\right) \sum_{n} c_{n}(t) n P(n) .
$$

Calling $a(t+1)=\sum_{n} c_{n}(t) n P(n)$ (that does not depend on $k)$, we have $c_{k}(t)=(k \tau) / z \exp \left(-J / k^{\alpha}\right) a(t)$ and thus

$$
c_{k}(t+1)=c_{k}(t) \frac{\tau}{z} \sum_{n} \exp \left(-\frac{J}{n^{\alpha}}\right) n^{2} P(n) .
$$

The critical boundary is therefore given by

$$
\sum_{k} \exp \left(-\frac{J_{c}}{k^{\alpha}}\right) k^{2} P(k)=\frac{z}{\tau}
$$

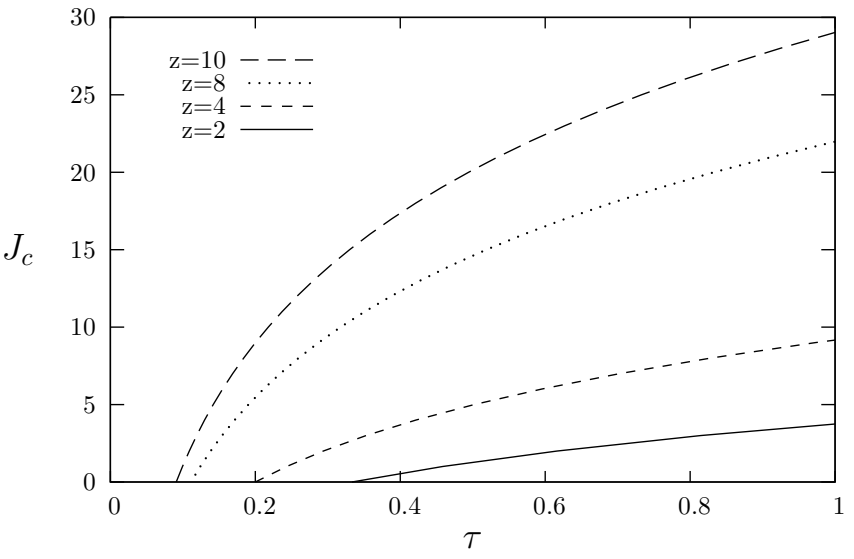

FIG. 3: The mean-field dependence of the critical value of precaution level $J_{c}$ with respect to the bare infectivity $\tau$ for Poissonian networks, with average connectivity $z$ and $\alpha=1$

from which one could obtain $J_{c}$ as a function of $\tau$ (we replaced $n$ by $k$ for consistency with the rest of the paper). In the case $J=0$ (no risk perception), the formula gives

$$
\tau_{c}=\frac{\mu_{2}}{z}=\frac{\left\langle k^{2}\right\rangle}{\langle k\rangle},
$$

which is a well-known result [9, 16].

In the case of fixed connectivity, $P(k)=\delta_{k z}$, and for $\alpha=1$

$$
J_{c}=z \log (\tau z)
$$

In the absence of perception $(J=0)$ one has $\tau_{c}=1 / z$.

For Poissonian networks (random graphs),

$$
P(k) \simeq \frac{z^{k} e^{-z}}{k !}
$$

Numerical integration of Eq. (3) for $\alpha=1$ gives the results shown in Figure 3 One can notice that for every value of $\tau$ and finite average connectivity $z$, there is always a value of the precaution level $J_{c}$ that leads to the extinction of the epidemics.

For non-assortative scale-free networks with exponent $\gamma, P(n) \propto n^{-\gamma}$, the sum in Eq. (3) diverges unless $\gamma>3$, irrespective of $\alpha$.

This implies that at the mean-field level, any level of precaution is not sufficient to extinguish the epidemics.

\section{NUMERICAL RESULTS}

The mean-field approximation disregards the effects of (correlated) fluctuations in the real system. Indeed, the effects of random and/or long-range connections may disrupt correlations. We found that the behavior of microscopic simulations with random rewiring, both in the quenched and annealed version, is well reproduced by 


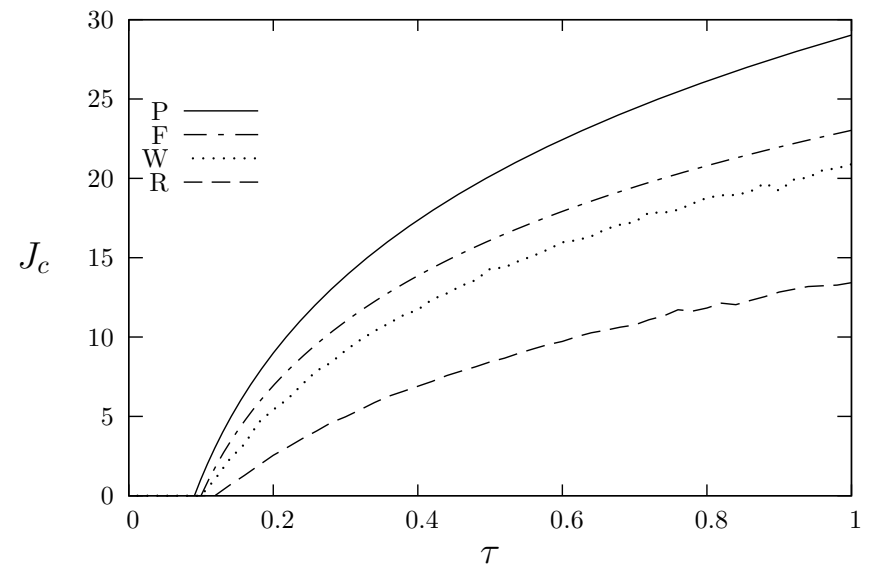

FIG. 4: Critical value $J_{c}$ of the precaution level as a function of the base infectivity $\tau$ average connectivity $k=10$ for the Poissonian mean-field (P), fixed connectivity mean Field, Eq. (4) $(\mathrm{F})$, and numerically $(N=1000)$, for the annealed rewired $p=1(\mathrm{~W})$ and regular one-dimensional $(\mathrm{R})$ cases.

mean field simulations with a white noise term, with amplitude proportional to $\sqrt{c(1-c) N}$. The noise term (or the fluctuations in microscopic simulations) may bring the infection to extinction if the average (or mean-field) oscillations come close to $c=0$, as is often the case for a choice of $J$ for which chaotic behavior appears in the mean-field approximation.

For regular (fraction of rewired links $p=0$ ) and rewired $(p>0)$ lattices, it is always possible to observe a continuous transition towards a critical level $J_{c}(\tau)$, such that the infection become extincted, for every value of the bare infectivity $\tau$, as shown in Figure 4.

For scale-free networks, we concentrated on the case illustrated in Section [II, which can be considered a worstcase scenario $(\gamma=2$, diverging second and first moments of input distribution).

Simulations show that for $\alpha=1$ (Eq. (2)), there is no value of $J_{c}$ for which the infection may be stopped (although not all population is always infected), for any value of $\tau$, in agreement with the mean-field analysis.

The investigation of nodes that are more responsible of the spreading of the infection reveals, as expected, that the nodes with higher input connectivity (hubs) stay ill most of time, Figure 5, Notice that also nodes with high input connectivity have finite output connectivity, so the above relation is not trivially related to the infection level.

In real life, however, public service workers who are exposed to many contacts (like medical doctors, for instance) use additional safety measures. In order to include this effect in the model, we use the parameter $\alpha$, Eq. (2), that up to now have been set to one. The effect of this parameter is to increase the perception of the risk (or the safety measures) for nodes with higher connectivity. As shown in Figure 6, as soon as $\alpha<1$, a finite critical value of $J_{c}$ appears. The transition from the

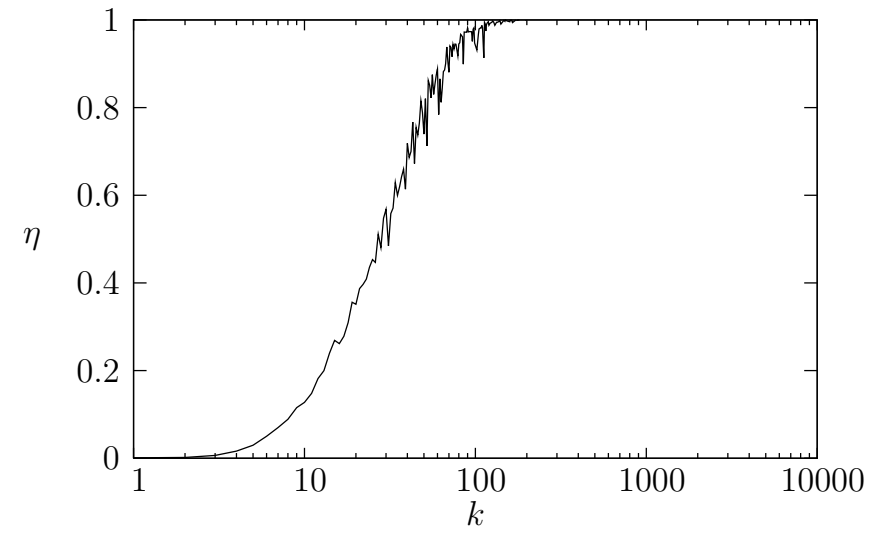

FIG. 5: Fraction of time spent ill $(\eta)$ in the scale-free case, as a function of $k$ for $K=10, J=10$.

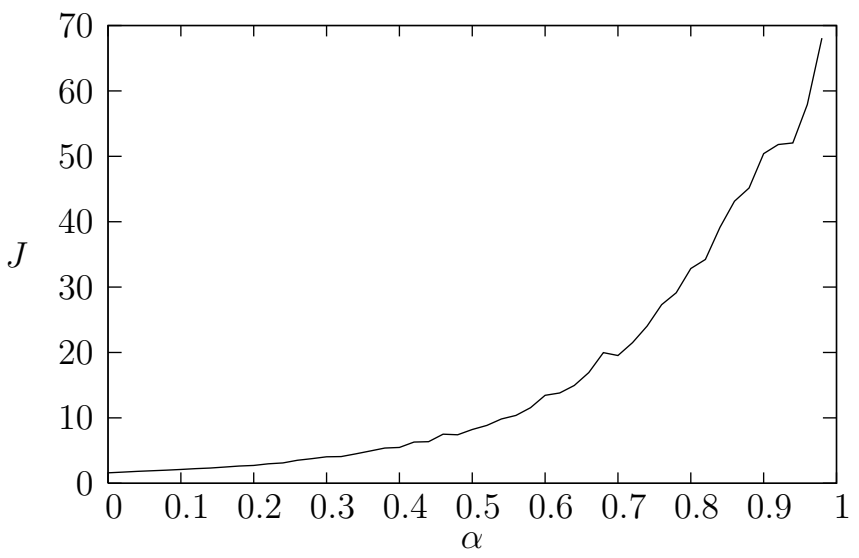

FIG. 6: Dependence of the critical value of the perception, $J_{c}$, as a function of the exposure-enhanced perception parameter $\alpha, K=4, \tau=1, N=10000$.

active $(c>0)$ to the absorbing $(c=0)$ state occurs suddenly, due to fluctuations. Essentially, nodes with high connectivity may fail to be infected due to their increased perception of the infection, and this stops efficiently the spreading. This effect is similar to targeted immunization, but is not captured by the mean-field analysis. It is a dynamical effect over a network far from the percolation threshold, and thus containing loops.

The transition may be a finite-size effect, related to the unavoidable cut-off in the degree distribution for finite populations, although simulations with populations from $N=5000$ up to $N=80000$ do not show any systematic change in the transition point.

\section{CONCLUSIONS}

In conclusion, we have studied the effects of risk perception in a simple SIS model for epidemics spreading. These effects are modulated by two parameters, $J$ and 
$\alpha$, that reduce the infectivity of the disease as a function of the fraction of people in the neighborhood that are manifestly ill. The first parameter modulates the linear response, while the second models non-linear effects like the increasing of prevention due to a public exposed role. We found that for fixed or peaked connectivity there is always a finite value $J_{c}$ of perception that makes the epidemics go extinct. We studied the evolution of the disease in a "worst case" social networks, with scale-free input connectivity and an exponent $\gamma \simeq 2$, for which both the average input connectivity and fluctuations diverge. In this case a linear perception cannot stop the disease, but we found that, as soon as the perception is increased in a non-linear way $(\alpha<1)$, the epidemics may get extincted by increasing the perception level. This latter transition is not continuous and is presumably induced by fluctuations in hubs. It may be due to the finiteness of population.

The mechanism that we propose is somehow analogous to vaccination of hubs, except that is is a dynamics effect due to the local level of diffusion of the disease, and is not exclusively related to local connectivity. We think that a similar mechanism is at the basis of the robustness of human population with respect to epidemics, even in the absence of immunization procedures. One may speculate if, in consequence of such robustness, humans have been selected to exhibit visual signs of the most common diseases, which certainly does not favors the spreading of infective agents. Another common symptom of an illness is the tendency to isolation, which again could be the result of selection.

\section{Acknowledgements}

L.S. research is supported by the EMBO organization under the contract ASTF 12-2007. Authors acknowledge fruitful discussions with F. Di Patti and A. Guazzini.
[1] V. Colizza, A. Barrat, M. Barthélemy and A. Vespignani, PNAS 103, 2015 (2006).

[2] D. J. Watts and S. H. Strogatz, Nature 393, 440 (1998).

[3] R. Pastor-Satorras, A. Vespignani, Phys. Rev. E 63, 066117 (2001).

[4] M. E. J. Newman, The Structure and Function of Complex Networks, SIAM REVIEW 45, 167 (2003).

[5] R. Albert and A-L. Barabasi, Rev. Mod. Phys. 74, 47 (2002).

[6] S. N. Dorogovtsev, J. F. F. Mendes and A. N. Samukhin, Phys. Rev. Lett. 85, 4633 (2000).

[7] In SIR dynamics the infective node cannot be reinfected, so $k$ is replaced by $k-1$.

[8] R.M. Anderson and R.M. May, Infectious Diseases of $\mathrm{Hu}$ mans: Dynamics and Control (Oxford University Press, Oxford, 1991).

[9] M. Boguña, R. Pastor-Satorras and A. Vespignani, Phys. Rev. Lett. 90, 028701 (2003).

[10] A. Vázquez and Y. Moreno, Phys. Rev. E 67, 015101(R) (2003).

[11] Y. Moreno, J.B. Gómez and A. F. Pacheco, Phys. Rev. E 68, 035103(R) (2003).

[12] V.M. Eguiluz and K. Klemm, Phys. Rev. Lett. 89, 108701 (2002).

[13] A. Vázquez, M. Boguna, Y Moreno, R. Pastor-Satorras and A. Vespignani, Phys. Rev. E 67, 046111 (2003).

[14] R. Cohen, S. Havlin and D. ben-Avraham, Phys. Rev. Lett. 91, 247901 (2003).

[15] R. D. Smith, Infectious Disease and Risk: Lessons from SARS, London: The Nuffield Trust, 2005.

[16] M. E. J. Newman, Phys. Rev. E 66, 016128 (2002).

[17] R. Olinky and L. Stone, Phys. Rev. E 70 030902(R) (2004).

[18] D.S. Callaway, M.E.J. Newman, S.H. Strogatz, and D.J.
Watts, Phys. Rev. Lett., 85, 5468 (2000).

[19] R. Cohen, K. Erez, D. ben-Avraham and S. Havlin, Phys. Rev. Lett. 85, 4626 (2000).

[20] S. Riley, C. Fraser, C. A. Donnelly, A. C. Ghani, L. J. Abu-Raddad, A. J. Hedley, G. M. Leung, L.-M Ho, T.-H Lam, T. Q. Thach et al., Science 300, 1961(2003).

[21] M. Lipsitch, T. Cohen, B. Cooper, J. M. Robins, S. Ma, L. James, G. Gopalakrishna, S. K. Chew, C. C. Tan, M. H. Samore et al. Science 300, 1966 (2003).

[22] L. Hufnagel, D. Brockmann and T. Geisel, PNAS 101, 15124 (2004).

[23] M. Kamo and A. Sasaki, Physica D 165, 228 (2002).

[24] R. Pastor-Satorras, A. Vespignani, Phys. Rev. Lett. 86, 3200 (2001).

[25] A.H. Eagly and S. Chaiken, The Psychology of Attitudes (Harcourt, Fort Worth, TX 1993). A.H. Eagly and S. Chaiken, Attitude structure and function, In D. T. Gilbert, S. T. Fiske and G. Lindzey (eds), The Handbook of Social Psychology, (McGraw-Hill, New York 1998) Vol. 1, pp. 269-322.

[26] P. Glendinning and L.P. Perry, J. Math. Biol. 35, 359 (1997).

[27] B. T. Grenfell, O.N. Biørnstad and B.F. Finkenstädt, Ecological Monographs 72, 185 (2002).

[28] L. Sguanci, P. Lió and F. Bagnoli, The influence of risk perception in epidemics: a cellular agent model, in Cellular Automata, S. El Yacoubi, B. Chopard and S. Bandini, editors (Lecture Notes in Computer Science LNCS 4173, Springer, Berlin 2006) pp. 321-329.

[29] N. Boccara, O.Roblin and M. Roger, Phys. Rev. E 50 4531 (1994).

[30] F. Bagnoli, F. Franci and R. Rechtman, Phys. Rev. E 71, 046108 (2005). 